\title{
Parallel Fringe Search Algorithm for VLBI Software Correlator Based on Computer Cluster
}

\author{
Yun Yu, Wei-Min Zheng, Ping Rui, Juan Zhang, \\ Feng-Xian Tong, Yong-Biao Shi \\ Shanghai Astronomical Observatory, Chinese Academy of \\ Sciences \\ E-mail: zhwm@shao.ac.cn
}

\author{
Wei-Min Zheng, Juan Zhang \\ Key Laboratory of Radio Astronomy, Chinese Academy of \\ Sciences, Nanjing, China \\ E-mail: zhwm@shao.ac.cn
}

\begin{abstract}
The Chinese VLBI Network correlator has been successfully adopted in the China's lunar exploration project for probe tracking. However, due to the probe orbit maneuver, sometimes the error of the predicted VLBI time delay can be quite large. The correlator fringe search module needs new methods to tackle the multi-processors environment parallel problem and to keep the real-time ability. This paper presents a high-speed parallel fringe search algorithm for the VLBI software correlator working on the cutting-edge computer cluster. Guided by the fringe search module, CVN software correlator accomplished the real-time data correlation and supported the first Chinese lunar surface soft landing in CE-3 mission.
\end{abstract}

Keywords: VLBI; correlator; fringe search; MPI; lunar exploration

\section{INTRODUCTION}

Very Long Baseline Interferometry (VLBI) is a radio interference technique, which was developed in the 1960s [1]. It combines several small radio telescopes in different places and adopts highly stable hydrogen maser to obtain high spatial resolution by using radio interference method [2].
VLBI has been widely adopted in astrophysics and astrometry, the Earth Orientation Parameters (EOP) measurement, and the deep space probe tracking and orbit determination [3].

In early 1970s, the U.S. National Aeronautics and Space Administration (NASA) Jet Propulsion Laboratory (JPL) scientists adopted VLBI in "Voyager 1" spacecraft navigation demonstration and achieved very good results. In the same year, the relative position of the "Appollo 16" lunar module and lander was firstly measured with the help of VLBI [5]. In 1985, the Soviet Union used VLBI to track the balloon released by VEGA spacecraft and to obtain the wind speed of different heights of the Venus atmosphere. In 2010, VLBA (Very Long Baseline Array) determined Cassini spacecraft Jupiter flight path, and the data accuracy is about 20 times higher than before. In 2011, Japanese used VLBI to determine the orbit of IKAROS (Interplanetary Kite-craft Accelerated by Radiation of the Sun). In 2014, ESA used VLBI to track the BepiColombo mercury detector. At present, VLBI has become the main technology of deep space high precision tracking.

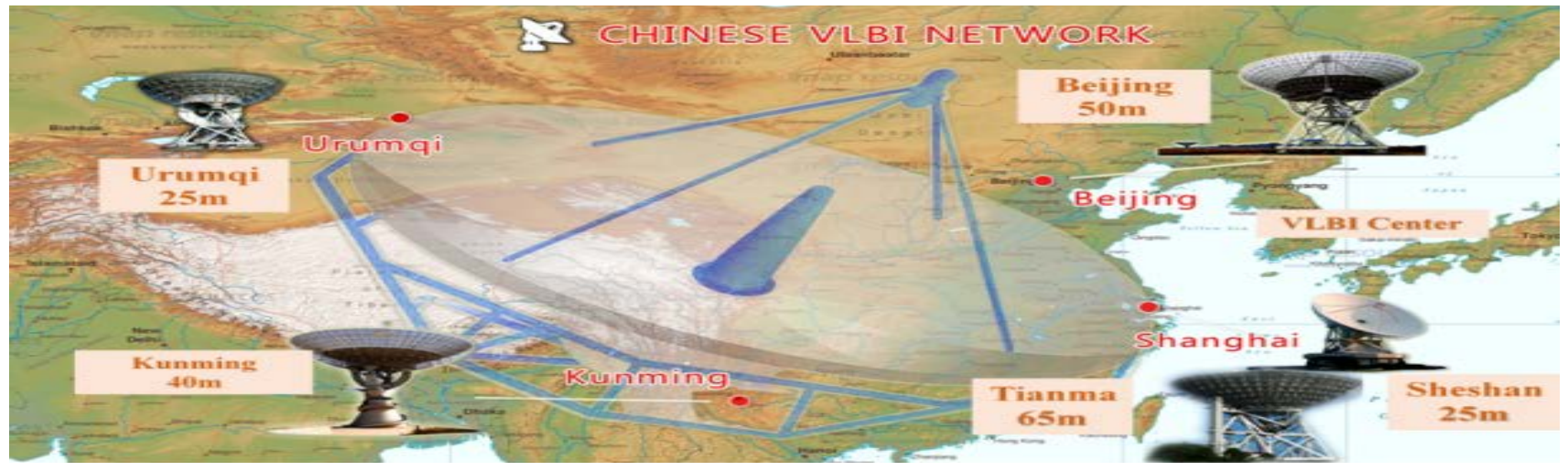

Figure 1. Chinese VLBI Network.

Chinese VLBI Network (CVN) consists of five radio telescopes: Beijing 50-m, Kunming 40-m, Urumqi 25-m Shanghai Sheshan 25-m, Shanghai Tianma 65-m and one VLBI data processing center as showed in Fig. 1. All of the data collected from those different stations are transmitted to the VLBI data center via high-speed network. Since
February 2003, CVN has completed a series of experiments on satellite observations, e.g., "Beidou" navigation satellite, TC-1 and SMART-1. In October 24, 2007, China launched the first lunar exploration satellite, Chang'e-1(CE-1). The CVN tracked the CE-1 during the transfer orbit and lunar orbiter mission [6]. From October 2010 to January 2013, the 
CVN provided accurate data to the Chang'e-2 lunar (CE-2) satellite. In December 2013, the CVN accomplished the orbit determination task of Chang'e-3 (CE-3) mission.

To tackle the high-performance computing problem in VLBI, fringe search is regarded as a promising method which provides high precision of time delay model for correlators. Development of fringe search for CVN initiated from tracking the lunar satellite. During the orbital maneuver phases, the accurate orbit element cannot be provided in advance, and so the correlator cannot work properly in such conditions. During recent years, the cluster system has been greatly developed in the field of high performance computing. It can deal with complex scientific computing and parallel processing of massive data. In addition, it has good cost-performance ratio and desirable scalability. Therefore, it is reasonable to conduct fringe search by using parallel arithmetic on the computer cluster.
In this paper, an algorithm of the fringe search is proposed and the associated structures based on the cutting-edge computer cluster are introduced. The algorithm performance is well verified in the Chang'e-3 (CE3) lunar exploration mission.

\section{FRINGE SEARCH ALGORITHM}

The VLBI data are recorded using Mark5B format. It is acquired using a base frequency of $84 X X M h z, 2 \mathrm{MHz}$ width, and 8 channels with 2-bit sampling. The aggregate data rate of every station is 64Mbps.

Fringe search firstly extracts the main carrier frequencies from the signals of different antennas to produce the group delay, and then compensates the delay and delay-rate. Fig. 2 shows the received spectrum of CE-3 satellite at X band of $\mathrm{BJ}$ and UR telemetry signals.

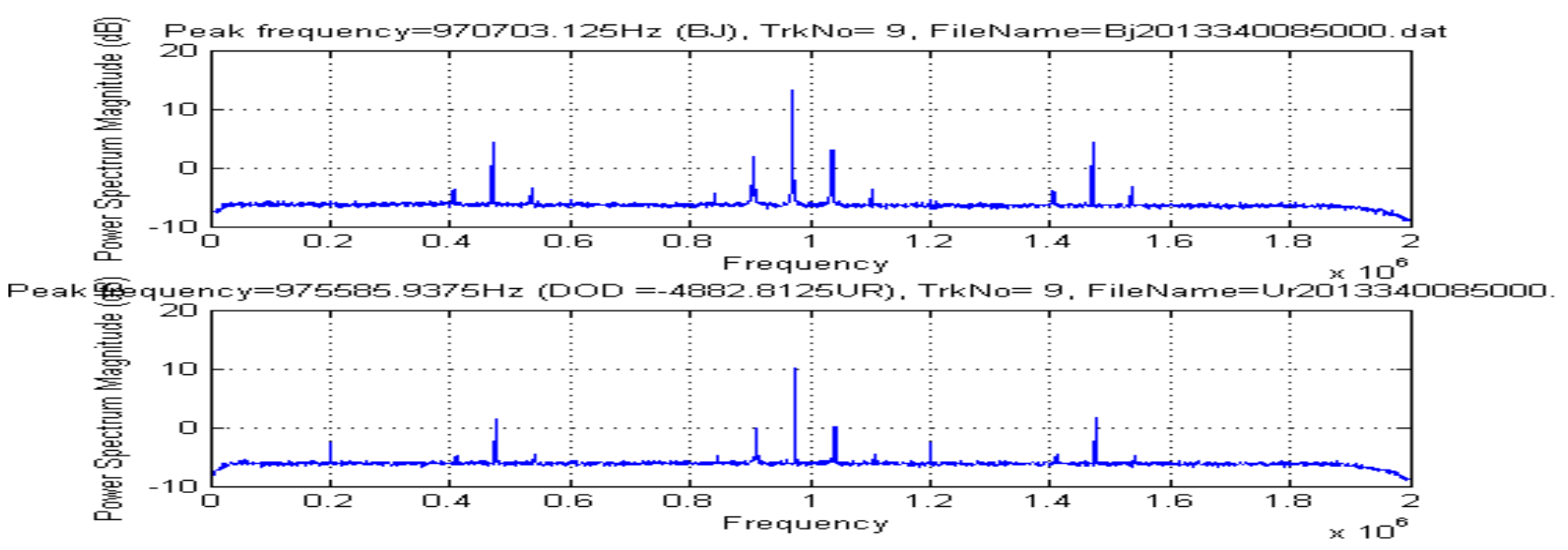

Figure 2. Auto spectrum of CE-3 satellite X band BJ and UR telemetry signals, concentrated around $1 \mathrm{MHz}$. The abscissa unit is frequency (MHz).

Fig. 3 depicts the algorithm flow chart in two-station case. When the fringe search works, the original observation data are sampled every 0.5 seconds. In 0.5 seconds, the time delay and delay rate are considered as constants. 


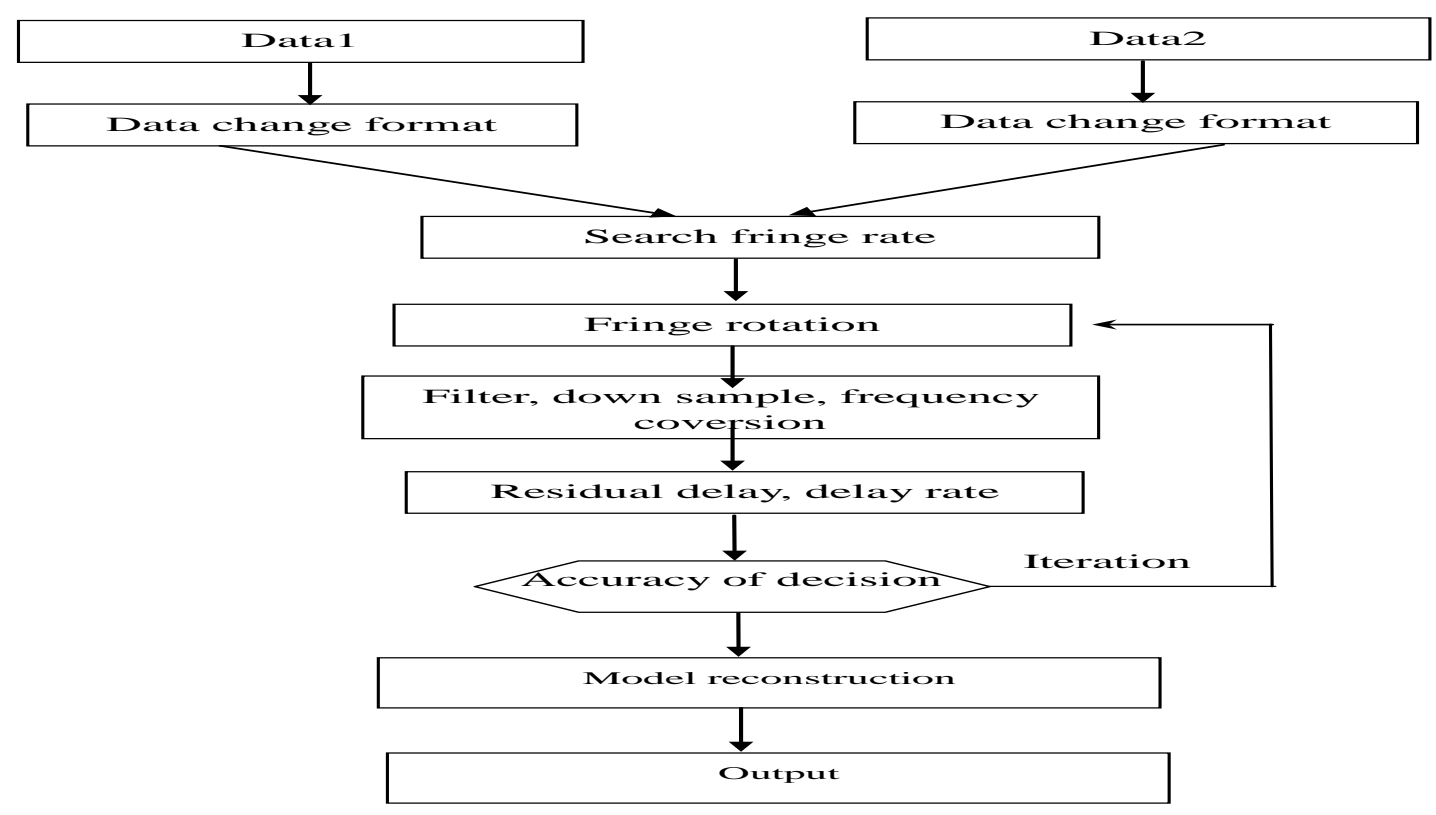

Figure 3. Algorithm of Fringe Search.

As depicted in Fig.3, the algorithm firstly searches the fringe rate to get the rough value, and then completes the fringe rotation. Since the telemetry signal's bandwidth is generally narrow and less than $500 \mathrm{KHz}$ and the bandwidth of raw data is about $2 \mathrm{MHz}$. The data is filtered by using a narrow filter then down sampled to compress the raw data [7]. Then the signal is converted to baseband signals through digital conversion.

The initial value of the time delay is obtained through the correlation function of the two station signal. And the value is used to make time delay compensation. Then the more accurate residual time delay is obtained from the phase of the cross correlation spectrum. Because the variance of the phase is inversely proportional to the square root of the signal to noise ratio, the method of weighted least square estimation is used to calculate the residual time delay [8].

In order to improve the accuracy of calculation, the iterative method is adopted. A delay reconstruction method is used to provide accurate time delay and delay rate to construct the processor satellite observation delay polynomial.

\section{IMPLEMENTATION OF PARALLEL FRINGE SEARCH}

In the satellite orbit maneuver case of s7b04a (Nov.5, 2007), it shows that frequency of doing one fringe search in one minute cannot correct the delay and the delay rate well, because the speed of the satellite in the orbit maneuver period changes quickly in one minute. Then the fringe search frequency increases to 10 points in every minute to get the better data fitting [9]. Fig. 4 shows the differences between the fringe search result and delay models of delay and delay rate of s7b04a. Four stations (Shanghai, Beijing, Kunming and Urumqi) formed six baselines (S-B, S-K, S-U, B-K, B-U, $\mathrm{K}-\mathrm{U})$. The re-constructed model is more close to the fringe search results and accurate enough for data correlations. 

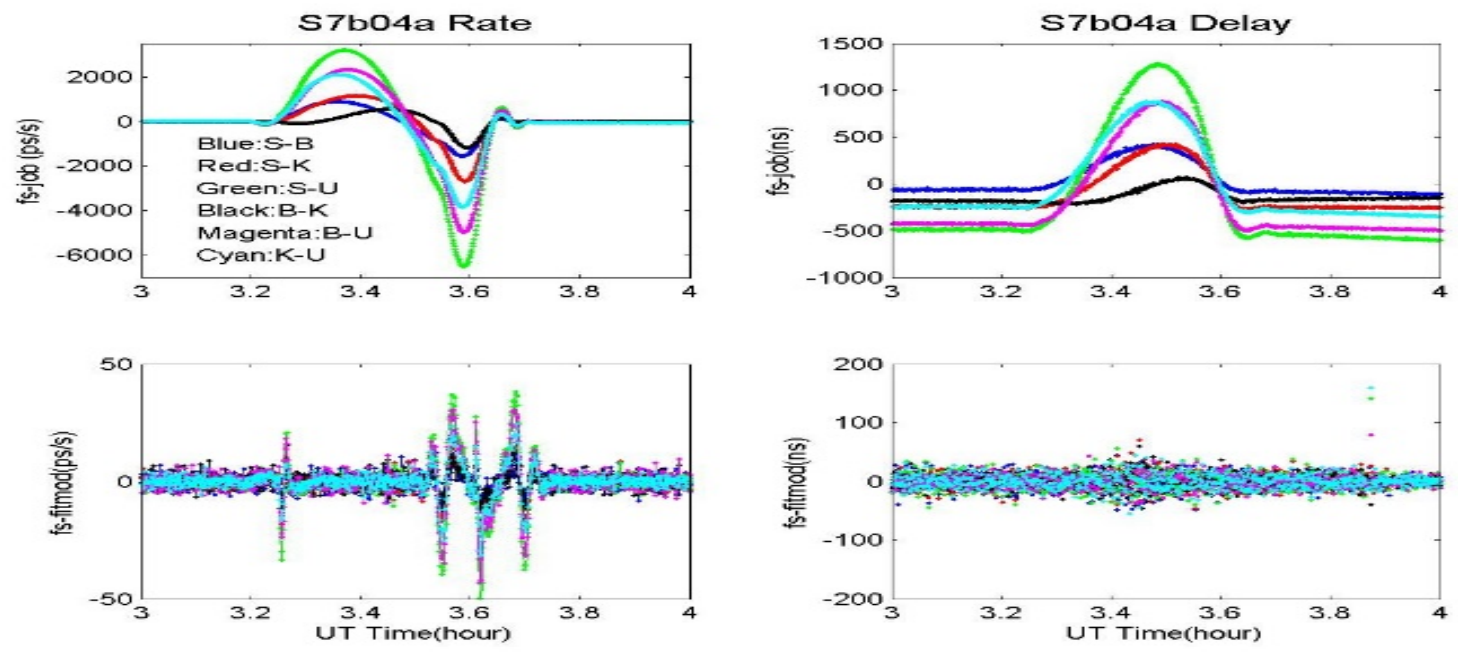

Figure 4. The fringe search data of 6 baselines during the lunar capture of the CE-1. Top: delay and rate differences between fringe search and a prior model. Bottom: delay and rate differences between fringe search and re-constructed model.

In CE-2 mission, the fringe search software running on PC servers consumes 15 seconds for each search operation. This speed cannot meet the requirements of the CE-3 project. After this, a new parallel fringe search module is written in C. Intel's Intel $\mathrm{C}++$ compiler, Intel Integrated Performance Primitive (IPP) and Open Multi-Processing (Open MP) are used to optimize the code. It only takes 3 to 4 seconds for each search operation. Although the performance has been greatly increased, the correlator needs 10 points to get the correct model using fringe search in real-time. So every scan data (5s) is split and processed in the cluster using MPI. Therefore, in CE-3 mission, the new fringe search software used the method of splitting the $5 \mathrm{~s}$ scan data to 10 segments and accomplishing the fringe search in every 0.5 s period.

Fig. 5 depicts the structure of the parallel fringe search software and the data flow. In the computer cluster, the parallel fringe search is managed by Node 0 , which reads and sends parameter files to others nodes. Data are loaded into memory from NFS by node1-10. These nodes are controlled by node 0 and process data. The data are received by node 0 and write to disk. They process the data in different nodes, and the delay and rate are sent back to node 0.

A model of a scan (every 5 seconds) is split to 10 segments in every 0.5 seconds, and four stations with 6 baselines have a total of 60 pieces of data. The cluster of fringe search has 16 nodes. The 60 segments of the data are processed in the 16 nodes. Each node processes about 4 pieces of data, and runs four processes.

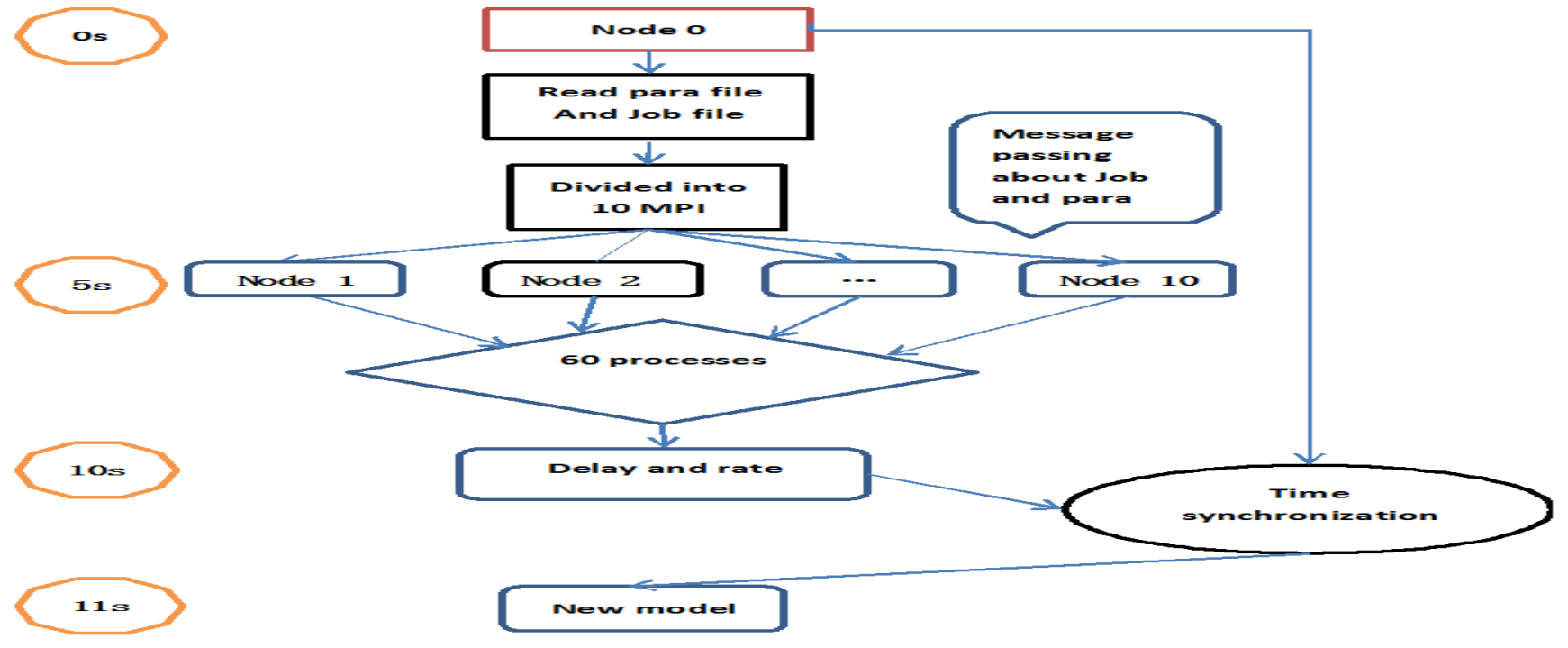

Figure 5. Structure of parallel fringe search. 
After braking, CE-3 probe entered into a $100 \mathrm{~km}$-high circular lunar orbit on 6 December 2013 at 09:53 UT. Fig. 6 is the difference of the fringe search delay and delay rate. We can clearly observe the delay and delay rate variations caused by the orbit maneuver. The time delay difference reaches about $5000 \mathrm{~ns}$, and the delay rate difference reaches about $80000 \mathrm{ps} / \mathrm{s}$.

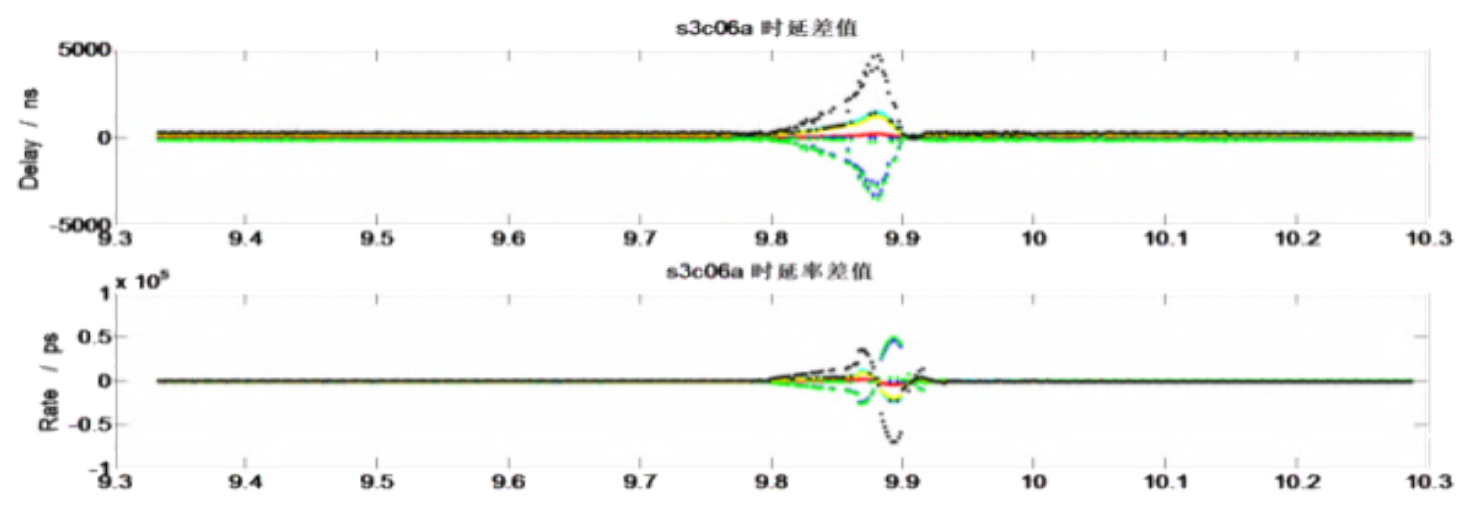

Figure 6. The difference of the fringe search delay and delay rate.

\section{PERFORMANCE EVALUATION}

In this section, we will elaborate the experimental setup and the achievement by using the cluster based fringe search method. The software environment is the Linux enterprise edition. Program uses C language, ICC compiler, the GNU software development kit. The MPI and Open MP libraries are also used to increase the computation speed.

The hardware platform is a commercial blade cluster depicted (Fig. 7). It has $5 \mathrm{I} / \mathrm{O}$ nodes (one quad-core CPU of E7-4820, 128GB memory, 300GB hard disk capacity), 16 compute nodes (6 CPUs/node, dual-core CPU of E5-2640, 32 GB memory, 300GB storage), and 2 management nodes (one dual-core CPU of E5-2620 24 GB memory, 900

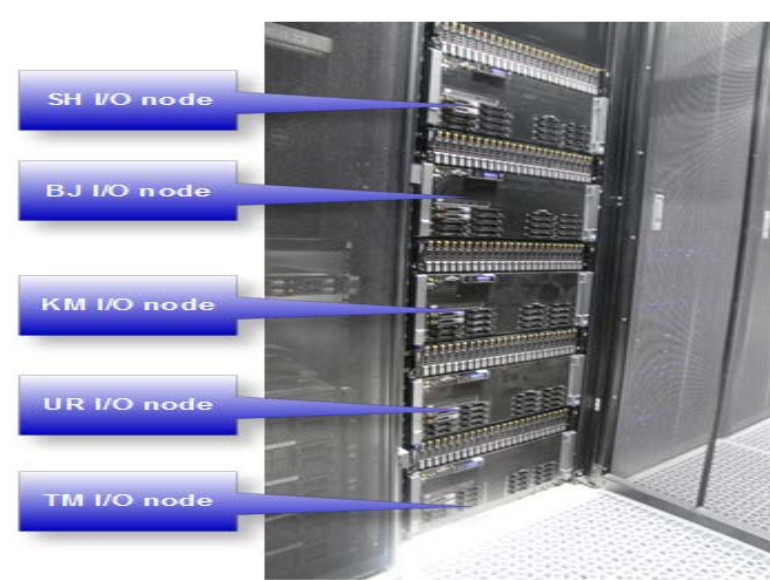

GB+214GB storage). The management network is 10G Ethernet, and the computer network is Infiniband.

We have evaluated if the processing time can meet the requirements. The CE-3 mission requires fringe search software to finish each search operation within 5 seconds. Table 2 presents the results of various nodes and the process under the situation. A single process program needs $15 \mathrm{~s}$ to do one fringe search operation, which is greater than $5 \mathrm{~s}$ data length. After adopting parallel optimization technique in the multiple processor environments, the speed has been greatly increased. The actual experimental results verify that the proposed method has greatly decreased the processing time and meet CE-3 mission real-time requirement.

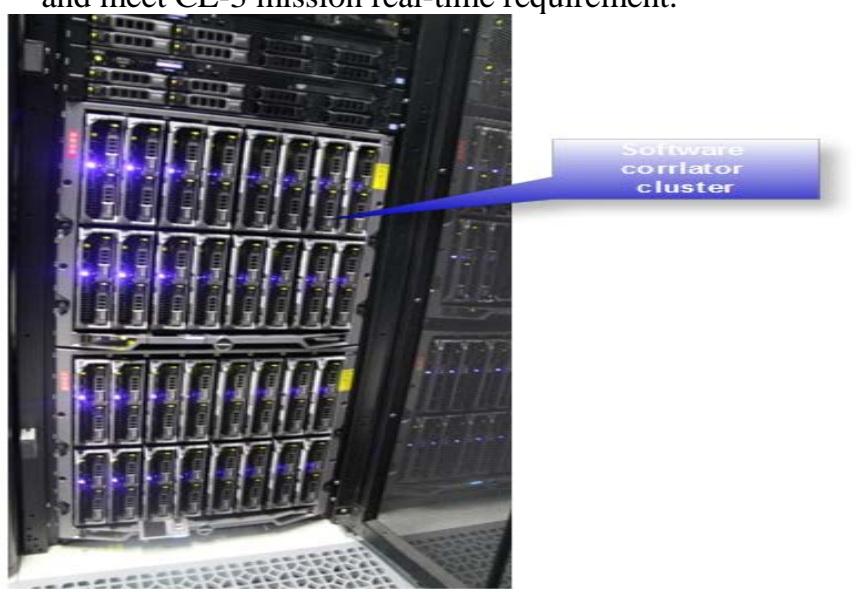

Figure 7. The fringe search cluster. Left: different nodes for different stations. Right: 32 nodes for the fringe search and the software correlator. 
TABLE I. TABLE PROCESSING TIME IN DIFFERENT NUMBER OF NODE AND PROCESS

\begin{tabular}{|c|c|c|c|}
\hline nodes & process & Data width $(\mathrm{MHz})$ & Processing time(s) \\
\hline 1 & 1 & 2 & 15 \\
\hline 10 & 60 & 2 & $3-4$ \\
\hline 10 & 120 & 2 & $2-3$ \\
\hline 20 & 120 & 2 & $1-2$ \\
\hline
\end{tabular}

The performance in terms of cross-correlation phase spectrum and amplitude spectrum is also evaluated. If the correlator uses the default predicted delay model, the correlator gets the wrong result (Fig. 8). In contrast, the correlator can produce correct results by using the Parallel fringe search program (Fig. 9). Specifically, it takes every five seconds to guarantee the real-time correlation in CE-3 mission.

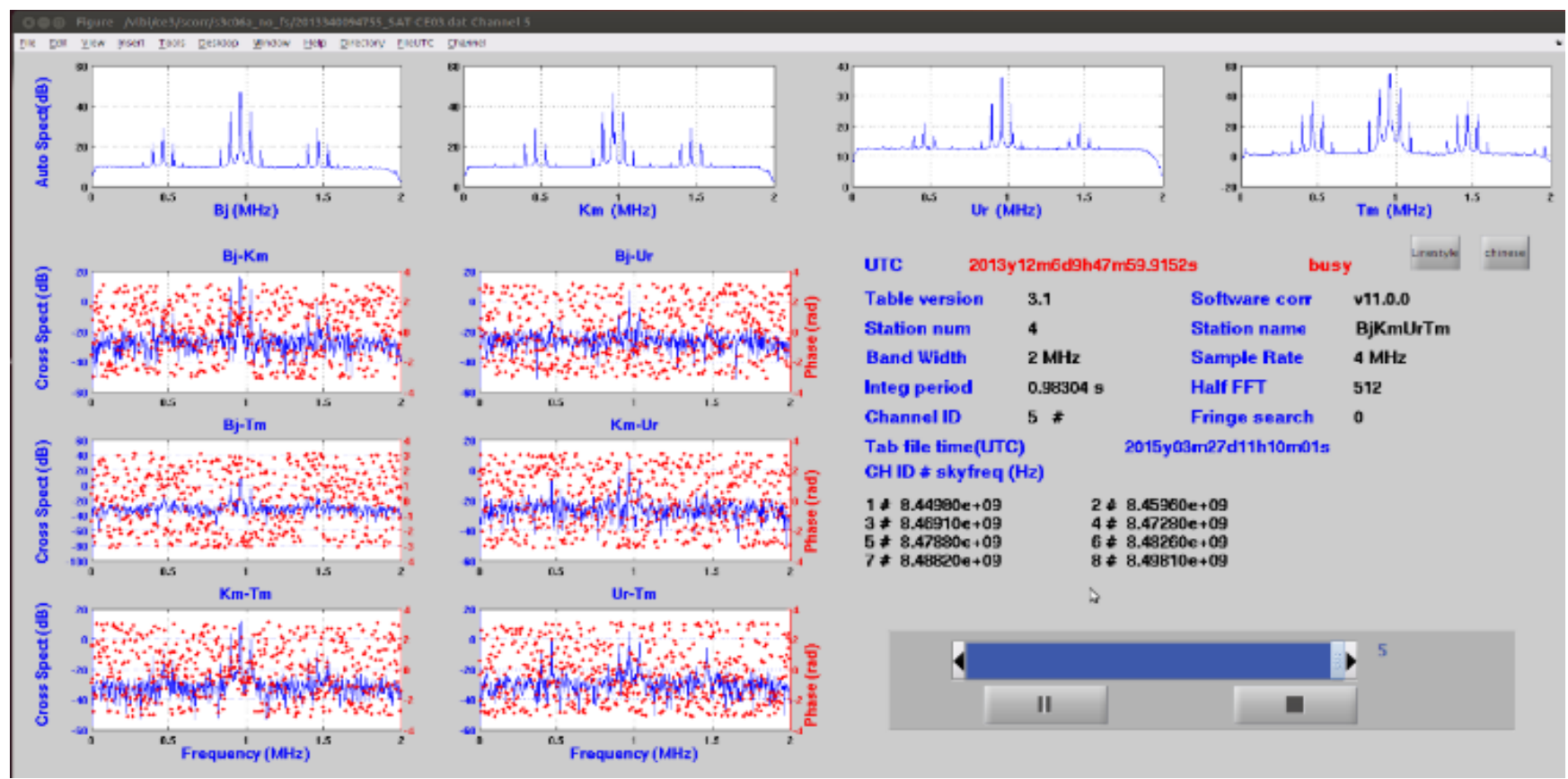

Figure 8. The cross-correlation phase spectrum (red points) and amplitude spectrum (blue points) of every baseline without using Parallel fringe search function from Quick Viewer GUI. The correlator gets wrong results.

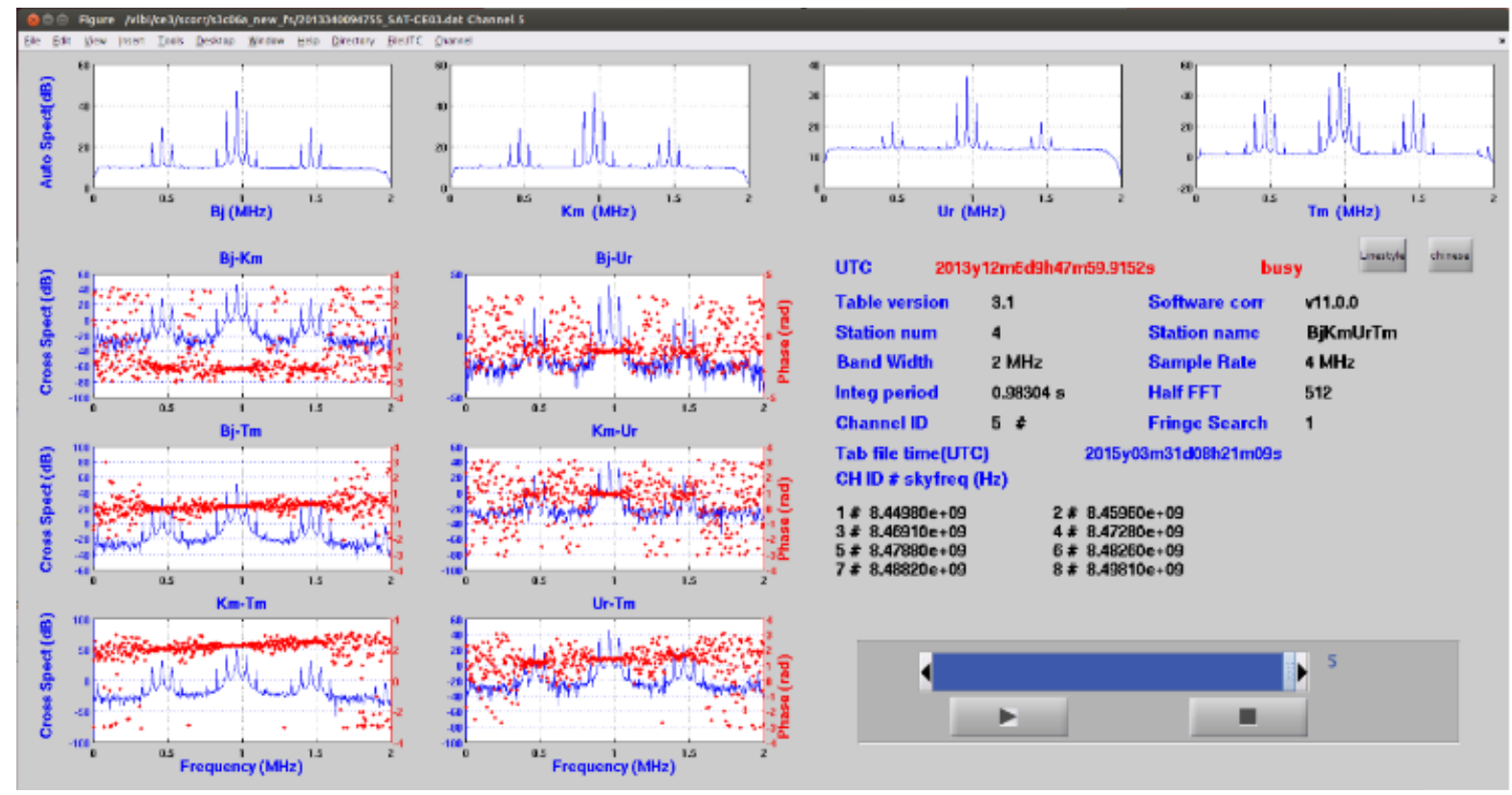

Figure 9. The cross-correlation phase spectrum (red points) and amplitude spectrum (blue points) of every baseline using the parallel fringe search function from Quick Viewer GUI. The correlator gets correct results. 


\section{CONCLUSION}

In this paper, a high-speed fringe search method based on the cutting-edge computer cluster is presented to solve the predicted VLBI time delay errors in the lunar orbit maneuver phase. The structure of the fringe search software and its applications in the CE3 lunar mission are elaborated in detail. Experimental results verify that this method can meet the real-time requirement.

\section{ACKNOWLEDGMENT}

This paper is sponsored by the Natural Science Foundation of China (11603062, 11373061, 11173052, 11573057, U1531116), the Shanghai Natural Science Foundation (15ZR1446800), the Science \& Technology Commission project of Shanghai Municipality (06DZ22101), the program of Shanghai Subject Chief Scientist (14XD1404300), CAS Key Technology Talent Program and the Chinese lunar exploration project. The authors appreciate the support of CVN data processing center, the help of Dr. Feng-chun Shu and Dr. Li Tong.

\section{REFERENCES}

[1] A R. Thompson, J M. Moran and G W. Swenson, "Interferometry and Synthesis in Radio Astronomy,” New York: John Wiley \& Sons, pp. 29-33,1986.
[2] A. Deller, W. Brisken, "software correlators in radio astronomy", SPIE Newsroom. DOI: 10.1117/2.1200903.1587

[3] A. Deller, W. Brisken, "DiFX2: A more flexible, efficient, robust and powerful software correlator", the Astronomical Society of the Pacific, Vol. 123, No. 901(March 2011), pp.257-385.

[4] W Zheng, "The Software Correlator of the Chinese VLBI Network", IVS 2010 General Meeting Proceedings, pp.157-161

[5] RW King, "Precision selenodesy via differential very-long-baseline interferometry", Thesis Massachusetts Inst of Tech, pp1-4, 1975

[6] J Ping, "Applications of New CVN for First Chinese Lunar Mission”, Cospar Scientific Assembly, 2008, 37:2447

[7] W Zheng, Z Chen "Parallel Software Correlator Based on Multi-Core PC Servers and Computer Cluster", International Conference on Information Science \& Engineering, 2009:303-306

[8] Zheng Weimin, Quan Ying, "The Software Correlator of the Chinese VLBI Network", Sixth International VLBI Service for Geodesy and Astronomy. Proceedings from the 2010 General Meeting, "VLBI2010: From Vision to Reality". Held 7-13 February, 2010 in Hobart, Tasmania, Australia., NASA/CP 2010-215864., p.157-161

[9] Shu Feng Chun, Yu Yun, Zheng Wei-Min, Zhang Xiu-Zhong, "Enhancement of satellite VLBI tracking system with capability of correlator model parameters prediction”, International Conference on Space Information Technology 2009. Proceedings of the SPIE, Volume 7651, article id. 76512F, 5 pp. (2009). 